The University of Maine

DigitalCommons@UMaine

Marine Sciences Faculty Scholarship

School of Marine Sciences

$2-1-2000$

\title{
Contumacious Beasts: A Story of Two Diastylidae (Cumacea) from Arctic Waters
}

\author{
S. Gerken
}

Les Watling

University of Maine - Main, watling@maine.edu

A. B. Klitgaard

Follow this and additional works at: https://digitalcommons.library.umaine.edu/sms_facpub

\section{Repository Citation}

Gerken, S.; Watling, Les; and Klitgaard, A. B., "Contumacious Beasts: A Story of Two Diastylidae (Cumacea) from Arctic Waters" (2000). Marine Sciences Faculty Scholarship. 28.

https://digitalcommons.library.umaine.edu/sms_facpub/28 


\title{
CONTUMACIOUS BEASTS: A STORY OF TWO DIASTYLIDAE (CUMACEA) FROM ARCTIC WATERS
}

\author{
Sarah Gerken, Les Watling, and Anne B. Klitgaard
}

\begin{abstract}
(SG, LW) Darling Marine Center, University of Maine, Walpole, Maine 04573, U.S.A. (SG e-mail: sgerke51@maine.edu); (ABK) Zoological Museum, University of Copenhagen, Universitetsparken 15, DK 2100 Copenhagen, Denmark
\end{abstract}

\section{A B S T R A C T}

\begin{abstract}
A full description of the subadult male holotype of the diastylid Ektonodiastylis robusta, new genus, new species, is presented, as well as of the adult male and adult female of E. nimia. Ektonodiastylis nimia is transferred from Brachydiastylis to Ektonodiastylis. The family definition of Diastylidae is expanded. The implications of this expansion on the systematics of the Cumacea in general, and Diastylidae and Gynodiastylidae in particular, are discussed.
\end{abstract}

A recent visit to the Zoologisk Museum, Copenhagen, by one of us (SG) included the opportunity to inspect material collected by a 1995 Danish marine biological expedition to Independence Fjord in northeastern Greenland. Independence Fjord is approximately $200 \mathrm{~km}$ long and $20 \mathrm{~km}$ wide at the maximum width, and covered with ice year-round. In the summer, long leads form in the ice due to tidal movements. The expedition to Independence Fjord, led by Dr. Eigeil Knuth, followed the framework of the Danish Peary Land Expeditions. The purpose of the expedition was to collect faunistic data for comparison with similar work done in the much smaller Jørgen Brønlund Fjord (Schiotte, 1989).

Within the Independence Fjord material, a species was found that appeared to fit the description of Brachdiastylis nimia Hansen, 1920, described from eastern Greenland; however, the males possessed only one pair of pleopods. Hansen's material did not include males, whereas the new specimens from northeastern Greenland include an abundance of subadult males and a single adult male, along with females of all stages. A single pair of pleopods was present in all males examined and, therefore, is not an instance of a single contumacious (=rebellious, disobedient) individual.

In addition, within a collection of isopods from the Canadian Arctic, another subadult male cumacean was discovered that also possessed a single pair of pleopods. Males with fewer than two pairs of pleopods are unusual in the family Diastylidae; in the genus Atlantistylis, pleopods are completely absent in males. The material described herein represents the first case of a male with one pair of pleopods.

\section{MATERIALS AND Methods}

Samples from Independence Fjord were collected with an Ockelmann detritus dredge through holes or leads in the ice, at depths of 20-200 m. The sample from the Canadian Arctic was obtained with a grab and had been stored in the collections of the Canadian Arctic Biological Station, Sainte Anne de Bellevue, Quebec. These collections have now been moved to the Canadian Museum of Nature in Ottawa. Drawings were prepared using a camera lucida mounted on a Wild compound microscope. Body length was measured from the tips of the pseudorostral lobes to the posterior border of the last body somite.

\section{SYSTEMATICS}

Family Diastylidae Bate, 1856

Diagnosis (modified after Day, 1980)._-Flagellum of antenna 2 of male with many short segments and reaching at least to posterior end of thorax. Mandible normally navicular (boat-shaped) but truncated above molar in Diastyloides. Branchial filament divided into numerous leaflets. Exopods present on maxilliped 3 and pereiopods $1-4$ in males, present in female on maxilliped 3 (except in Paradiastylis) and on pereiopods 1-2, rudimentary on or absent from pereiopods 3 and 4. Male with no pleopods (Atlantistylis), one (Ektonodiastylis) or two pairs of pleopods (all other genera); no outer process on inner ramus of pleopod. Telson variable, usually large, often with long postanal part, or short 

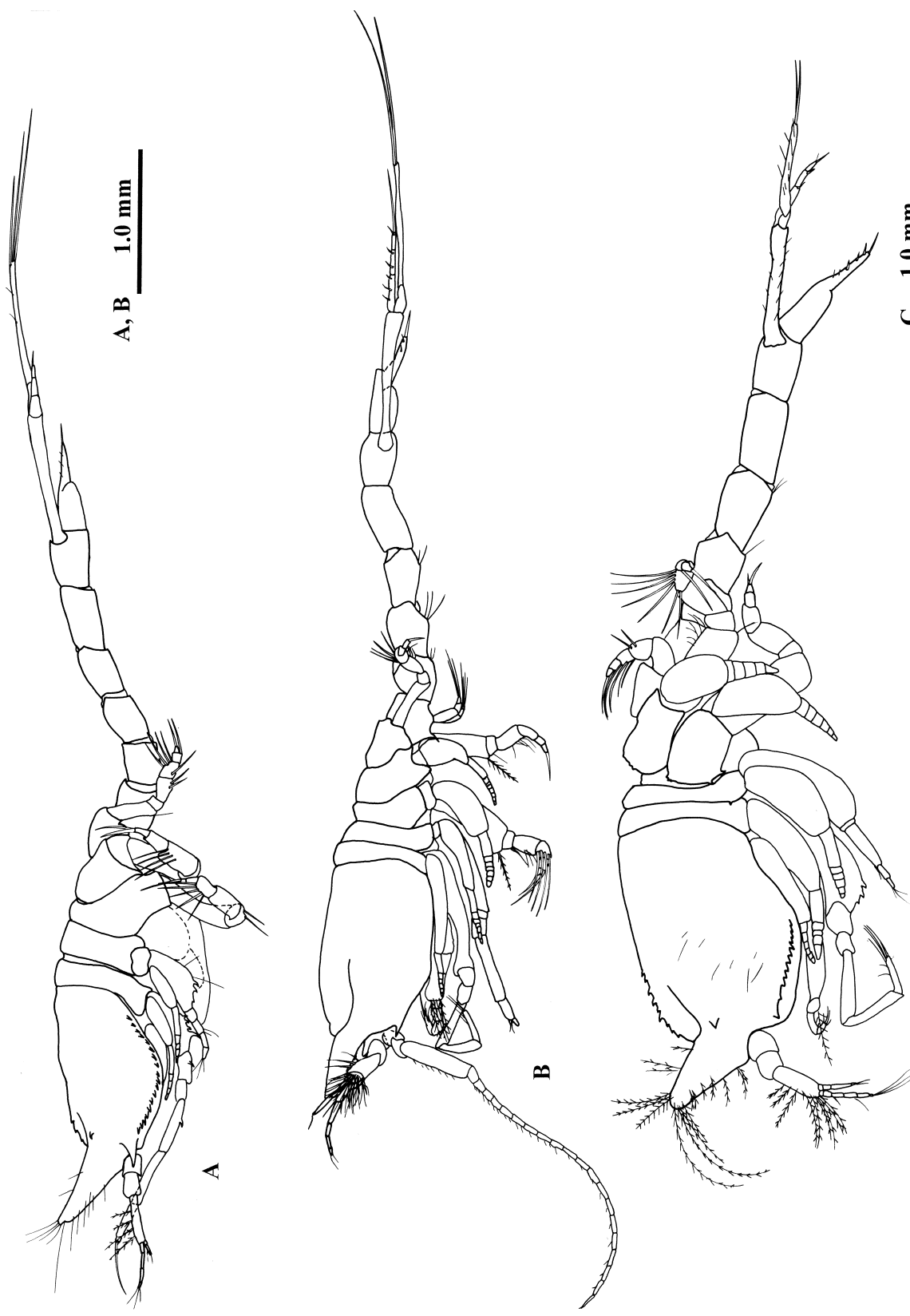

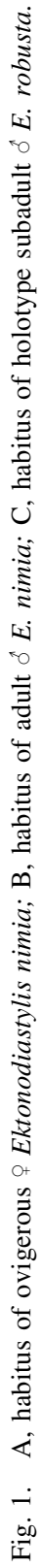


and poorly armed; bearing one pair of terminal setae or none. Uropods usually long and slender, endopod 1-3-segmented.

\section{Ektonodiastylis, new genus}

Type Species.-Ektonodiastylis nimia is designated as the type species; complete descriptions of both ovigerous + and adult $\delta$ are provided herein. Type material is that deposited by Hansen (1920). However, it should be noted that his syntypes are in poor shape.

Diagnosis.-Male with 1 pair of pleopods, pereiopod 2 dactyl minute and attached proximally to distal end of propodus.

Etymology.-The generic name is a combination of ektonos, Greek, meaning out of tune, and diastylis, from the family Diastylidae.

Other Species.-Ektonodiastylis robustus, new species.

Remarks.-This genus is very similar to Brachydiastylis, distinguishable at present only on male characters, primarily the possession of a single pair of pleopods. The peculiar arrangement of the dactyl and propodus on pereiopod 2 is also unusual and seems derivable from the condition seen in Brachydiastylis, namely, the extension of the propodus forms a large fixed finger, but in $E k$ tonodiastylis the dactyl is reduced to a small article that most likely is not movable, since there is no associated musculature in the propodus. Hansen (1920) noted the unusual pereiopod 2 in the male of Brachydiastylis resima and presumed it would also occur in his new species $B$. nimia, but he had no mature males. The other species of Brachydiastylis, B. hexaceros Lomakina, 1952, is being left in the genus for the time being, since the mature male is not known.

\section{Ektonodiastylis nimia (Hansen, 1920),} new combination

Material Examined.-Syntypes: 2 ovigerous ㅇ (ZMUC CRU-3437) "North of Steward Land," $70^{\circ} 30^{\prime} \mathrm{N}$. Additional material examined: 41 ovigerous $\circ \circ, 94$ subadult $\circ \circ, 1$ adult ô, 73 subadult ôे (ZMUC CRU-3573), Independence Fjord, Greenland, 4 August 1995, $82^{\circ} 07.06^{\prime} \mathrm{N}, 29^{\circ} 51^{\prime} \mathrm{W}, 35-40 \mathrm{~m}$.
Diagnosis.-Carapace produced as large tooth at anteroventral corner, pseudorostral lobes directed nearly horizontally, pereionites 3 and 4 directed posteroventrally, telson shorter than uropod peduncles, uropod exopod length greater than twice endopod length. Adult individuals near $3 \mathrm{~mm}$ in length.

Description.-Adult male. Length $3.3 \mathrm{~mm}$. Pseudorostral lobes 0.6 times carapace total length, bearing many plumose setae, eye lobe 0.1 times carapace length, with no lenses; carapace produced as large spine at anteroventral corner, otherwise smooth; antennal notch oblique (Fig. 1B).

Antennule stout, extending beyond pseudorostral lobes; peduncle articles 1 and 2 together slightly longer than article 3 , unarmed; peduncle article 3 dorsal margin bearing 4 long stout plumose setae, 2 small simple setae on distal margin; main flagellum of 6 articles, article 1 expanded and bearing many slender simple setae, 3 terminal articles bearing several simple setae; accessory flagellum of 4 articles, extending to distal margin of main flagellum article 3 , bearing few simple setae terminally (Fig. 2A).

Antenna long, extending past pereion but not extending to end of pleon; peduncle article 3 bearing 2 plumose setae, twice as long as article 4; article 4 unarmed; article 52.5 times as long as article 3, anterior margin bearing 6 groups of short simple setae; flagellum of 20 articles, articles decreasing in width and increasing in length distally, each article bearing 2 or 3 rows of small slender setae (Fig. 2B).

Mandibles, maxillulae, maxillae, maxilliped 1, and maxilliped 2 as in female.

Maxilliped 3 basis longer than all other articles together, 3 times as broad as merus, medial and lateral margins lined with fine hairlike setae, bearing 2 plumose setae medially, distolateral corner bearing 3 stout plumose setae, distomedial corner produced as 2 teeth; ischium compressed, unarmed; merus subequal to carpus, bearing 1 plumose seta laterally; carpus bearing 2 plumose setae distally; propodus and dactyl subequal, propodus bearing 2 sparsely plumose setae medially, 1 small plumose seta at distolateral corner; dactyl bearing 6 simple setae apically; exopod shorter than basis, basal article unarmed, flagellum bearing 10 long, stout plumose setae (Fig. 2C). 


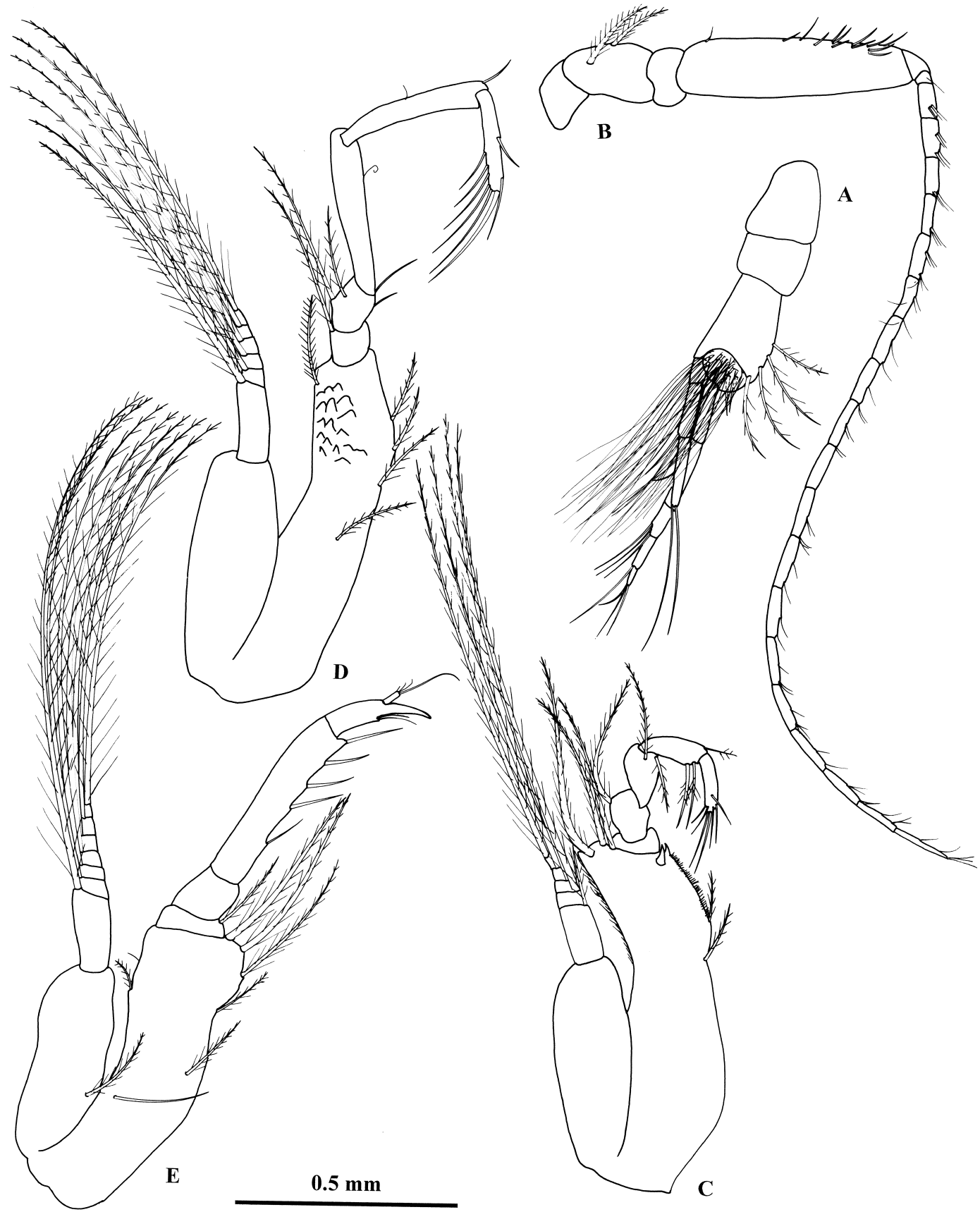

Fig. 2. Ektonodiastylis nimia adult $\mathrm{o}$. A, antennule; B, antenna; C, maxilliped 3; D, pereiopod 1; E, pereiopod 2.

Pereiopod 1 basis as long as next 4 articles together, posterior margin bearing 4 plumose setae, anterior margin bearing 1 plumose seta distally; ischium and merus subequal, ischium anterior margin bearing 1 plumose seta; merus anterior margin bearing 2 plumose setae; carpus and propodus subequal and unarmed; dactyl shorter than propodus, bearing 3 simple setae apically and 4 simple setae marginally; exopod as long as 
basis and ischium together, basal article unarmed, flagellum bearing 12 long, stout plumose setae (Fig. 2D).

Pereiopod 2 basis slightly longer than next 3 articles together, anterior margin bearing 1 plumose seta, posterior margin bearing 5 long plumose setae; ischium compressed, bearing 1 plumose seta at distal posterior corner; merus 3 times ischium length, unarmed; carpus 4 times merus length, posterior margin bearing 5 simple setae; propodus one-third carpus length, extended distally as fixed finger beyond insertion of dactyl, ridge at base of fixed finger bearing 3 small setae; dactyl minute, bearing several slender simple setae; exopod extending beyond merus, basal article bearing 1 plumose seta, flagellum bearing 14 long, stout plumose setae (Fig. 2E).

Pereiopod 3 basis longer than all other articles together, anterior margin with 2 small setae, bearing 1 plumose and 1 simple setae distally; ischium one-third length of merus, bearing 1 plumose seta anteriorly; merus anterior margin having 4 stout and 2 slender simple setae; carpus one-half merus length, bearing 7 stout annulate setae, increasing in length distally; propodus subequal in length to carpus, with 1 long, stout annulate seta distally; dactyl two-thirds propodus length, bearing 1 slender simple seta marginally, 2 stout simple setae terminally; exopod as long as basis, ischium, and merus together, basal article unarmed, flagellum bearing 12 long, stout plumose setae (Fig. 3A).

Pereiopod 4 basis about as long as all other articles together, distal anterior corner bearing 1 plumose seta, posterior margin bearing 1 pedunculate seta $\mathrm{A}$; ischium anterior margin bearing 2 plumose setae; merus as long as next 3 articles together, anterior margin with 4 short annulate setae; carpus as long as propodus and dactyl together, bearing 7 long, stout annulate setae, increasing in length distally; propodus and dactyl subequal in length, propodus bearing 1 long, stout annulate seta distally, 1 pedunculate seta A distally; dactyl bearing 1 slender simple seta marginally, 2 stout simple setae terminally; exopod longer than basis and ischium together, basal article unarmed, flagellum bearing 14 long, stout plumose setae (Fig. 3B).

Pereiopod 5 basis as long as next 3 articles together, bearing 2 simple and 2 plumose setae distally, 1 pedunculate seta marginally; ischium half length of merus, bearing 1 plumo-annulate seta distally; merus bearing 2 annulate setae marginally; carpus as long as propodus and dactyl together, with 4 annulate setae; propodus subequal to dactyl, bearing 1 stout annulate seta and 1 slender plumose seta; dactyl bearing 1 slender seta marginally and 2 stout simple setae terminally (Fig. 3C).

One pleopod pair present; pleopod basal article 3.5 times length of rami, medial margin bearing 2 simple and 2 long plumose setae; medial ramus uniarticulate, bearing 4 long, stout plumose setae apically; lateral ramus biarticulate, bearing 2 long, stout plumose setae terminally (Fig. 3D).

Telson 1.9 times length of pleonite 6, preanal section longer than postanal section, preanal section unarmed, postanal section lateral margins bearing 1 pair of slender setae and 2 pairs of stout setae, 1 pair of long microplumose setae terminally, stout setae short with 1 subapical setule (Fig. 3E).

Uropod peduncles longer than telson, shorter than exopods, medial margins bearing 5 stout microplumose setae; endopod 0.55 times length of exopod, of 3 articles, article 1 longest, medial margin bearing 3 stout microplumose setae, article 2 medial margin bearing 2 stout microplumose setae, article 3 medial margin with 1 stout microplumose seta, lateral margin bearing 1 small simple seta, and with 1 very long microplumose seta terminally; exopod of 2 articles, article 10.25 times length of article 2, unarmed, article 2 lateral margin bearing several small simple setae, and with 3 extremely long, microplumose setae terminally (Fig. 3E).

Ovigerous female. Length $3.7 \mathrm{~mm}$. Carapace with several teeth on anterior portion of dorsal crest, pseudorostral lobes 0.5 times total carapace length, bearing many setae, 1 tooth below pseudorostral lobe, carapace anteroventral corner produced as large tooth, antennal notch oblique, ventral margin lined with teeth, eyelobe not present; pereionites 1 and 2 free and not covered by carapace, pereionites $3-5$ produced posteroventrally, large separation between pereionites 2 and 3 ventrally (Fig. 1A).

Antennule extending past pseudorostral lobes; article 11.6 times as long as article 2, mediodistal corner produced as tooth, otherwise unarmed; article 2 bearing 1 pedunculate seta A medially; article 3 longer than articles 1 and 2 together, dorsal margin bearing 4 plumose setae, few small simple setae, 

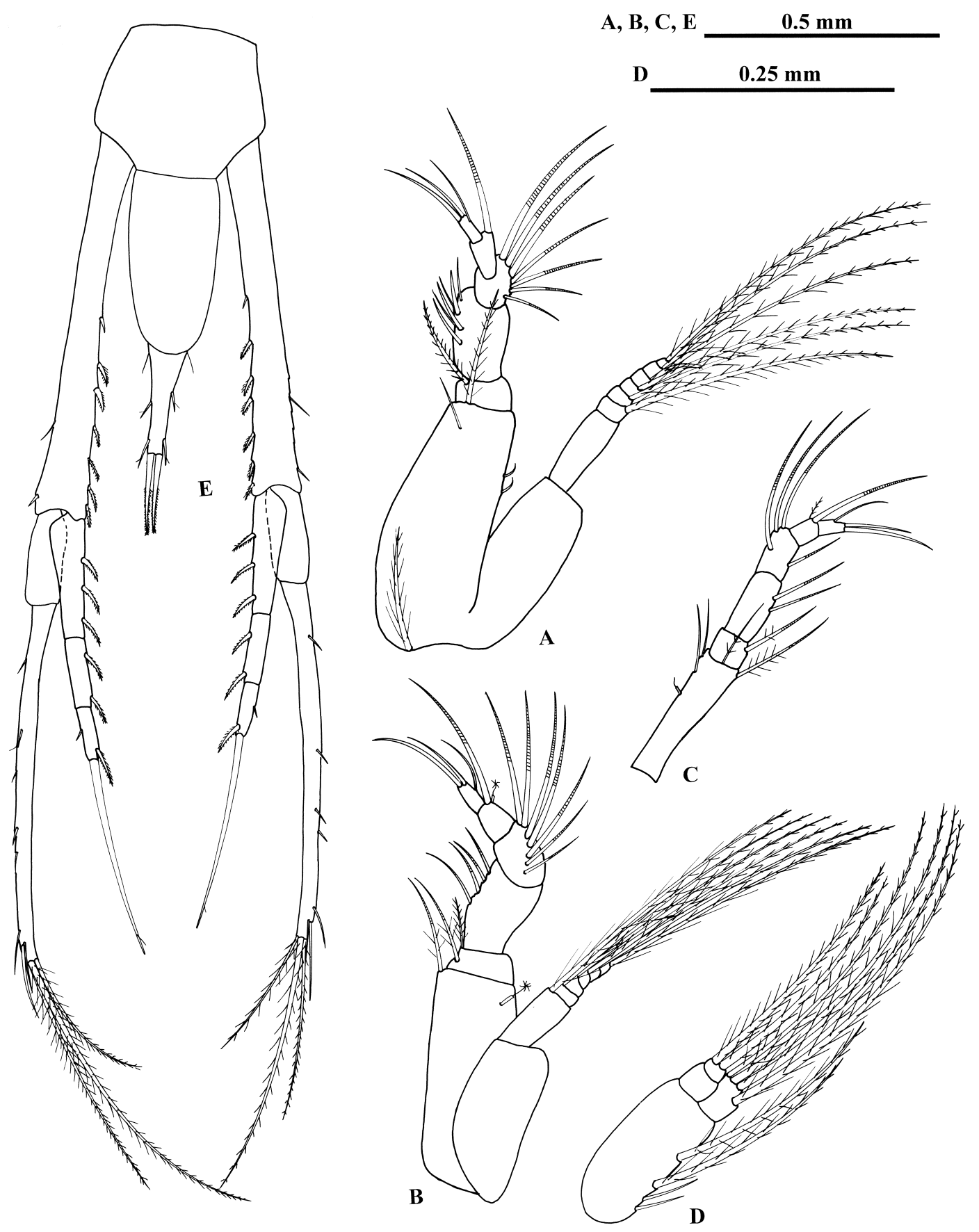

Fig. 3. Ektonodiastylis nimia adult ô. A, pereiopod 3; B, pereiopod 4; C, pereiopod 5; D, pleopod; E, telson and uropods.

laterodistal corner bearing 1 pedunculate seta; main flagellum of 4 articles, bearing several simple setae terminally; accessory flagellum of 1 article, slightly longer than article 1 of main flagellum, with few simple setae terminally (Fig. 4A).

Antenna rudimentary, of 3 articles, bearing 1 plumose seta terminally (Fig. 4B). 


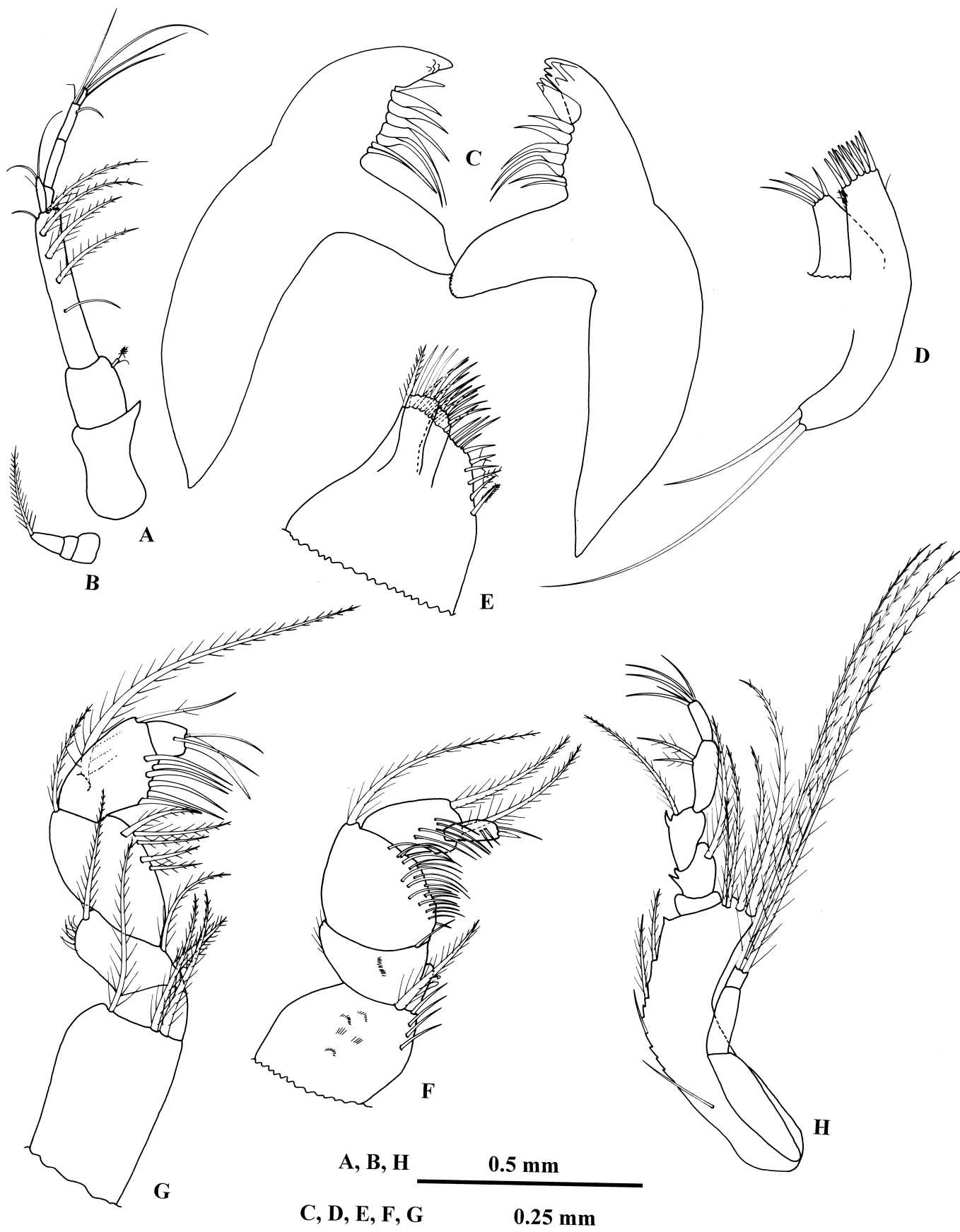

Fig. 4. Ektonodiastylis nimia ovigerous +. A, antennule; B, antenna; C, mandible; D, maxillule; E, maxilla; F, maxilliped 1; G, maxilliped 2; H, maxilliped 3.

Mandible navicular, lifting row of 7 setae; left mandible with stout, tricuspidate lacinia mobilis, incisor with 6 cusps; right mandible with slender lacinia mobilis, incisor with 5 cusps (Fig. 4C).
Maxillule of 2 lobes, broad lobe lateral margin bearing 1 simple seta, medial margin with many fine hairlike setae, distal margin bearing 2 rows of 5 stout setae; narrow lobe distal margin having 4 setae of varying 
lengths; palp bearing 2 long setae (Fig. 4D). Maxilla with 3 lobes, broad lobe distolateral corner bearing 1 plumose seta, distal margin with many simple setae, 1 pappose seta at distomedial corner, medial portion of lateral margin bearing several sparsely plumose setae; narrow lateral lobe bearing 5 simple setae terminally; medial narrow lobe bearing 3 or 4 simple setae terminally (Fig. 4E).

Maxilliped 1 basis medial margin bearing several simple setae and 1 pappose seta distally, produced as lobe medially, lobe bearing 2 hooks, anterior face with several ranks of small fine hairlike setae; ischium not present; merus not as long as carpus, bearing few simple setae; carpus distolateral corner bearing 1 long plumose seta, medial face carrying many simple setae; propodus bearing 2 plumose setae distally, medial face bearing many simple setae; dactyl one-third width of propodus, not as long as propodus, bearing 3 simple setae terminally (Fig. 4F).

Maxilliped 2 basis distal margin bearing 3 plumose setae; ischium unarmed; merus with 1 plumose seta at each distal corner, distolateral margin lined with fine hairlike setae, face bearing several rows of fine hairlike setae; carpus as long as propodus and dactyl together, distolateral corner with 1 plumose seta, medial margin bearing 5 plumose setae; propodus lateral margin with 2 plumose setae, medial margin bearing 6 simple setae; dactyl less than one-half length of propodus, bearing 3 slender and 1 very stout simple setae apically (Fig. 4G).

Maxilliped 3 basis longer than all other articles together, medial margin bearing several plumose and 1 simple setae, distolateral corner bearing 4 long, stout plumose setae, medial margin produced as several teeth/scales; ischium compressed, unarmed; merus produced as 2 teeth medially, distolateral corner bearing 1 plumose seta; carpus longer than merus, produced as 1 tooth medially, distomedial corner bearing 1 plumose seta; propodus subequal to carpus, with 2 plumose setae medially; dactyl two-thirds carpus length, bearing 6 simple setae terminally; exopod shorter than basis, basal article unarmed, flagellum bearing 4 long, stout plumose setae (Fig. 4H).

Pereiopod 1 basis longer than next 2 articles together, posterior margin produced as 2 large teeth, bearing 3 plumose setae, anterior distal margin bearing 3 plumose setae, ar- ticle covered with scales; ischium two-thirds merus length, posterodistal corner bearing 1 plumose seta; merus unarmed; carpus and merus subequal in length, bearing 1 simple seta; propodus bearing 3 simple setae; dactyl one-half length of carpus, bearing 6 simple setae marginally, 3 long simple setae terminally; exopod longer than basis and ischium together, basal article anterior margin produced as 5 teeth, otherwise unarmed, flagellum bearing 6 long, stout plumose setae (Fig. $5 \mathrm{~A})$.

Pereiopod 2 reduced, basis slightly longer than next 4 articles together, posterior margin produced as 2 teeth, bearing 1 simple and 3 plumose setae, anterior margin bearing 1 simple seta; ischium compressed, posterior margin bearing 1 plumose seta; merus one-half carpus length, posterodistal corner bearing 1 simple seta; carpus posterodistal corner with 2 simple setae; propodus not as long as carpus, posterodistal corner bearing 1 simple seta; dactyl not as long as propodus, bearing 1 simple seta marginally, 3 simple setae terminally; exopod about as long as endopod, basal article unarmed, flagellum with 6 long, stout plumose setae (Fig. 5B).

Pereiopod 3 basis as long as all other articles together, posterior margin produced as 1 large tooth, bearing 4 plumose and 1 plumoannulate setae, anterior margin bearing 3 simple setae; ischium 0.25 times length of merus, bearing 2 annulate setae; merus with 4 short annulate setae marginally; carpus one-half merus length, anterior margin bearing 7 stout annulate setae, increasing in length distally; propodus one-half length and one-half width of carpus, bearing 1 stout annulate seta and 1 pedunculate seta A distally; dactyl bearing 1 slender simple seta marginally, with 2 stout simple setae terminally; exopod not present (Fig. 5C).

Pereiopod 4 basis as long as next 3 articles together, posterior margin bearing 5 plumose setae, anterior margin bearing 2 slender simple setae, 2 pedunculate setae $A$; ischium 0.25 times length of merus, posterior margin bearing 2 annulate setae; merus posterior margin having 5 annulate setae; carpus less than one-half length of merus, anterior margin bearing 7 stout annulate setae, increasing in length distally; propodus slightly shorter than carpus, one-half width of carpus, bearing 1 annulate seta distally; dactyl onehalf length of propodus, bearing 1 slender seta 


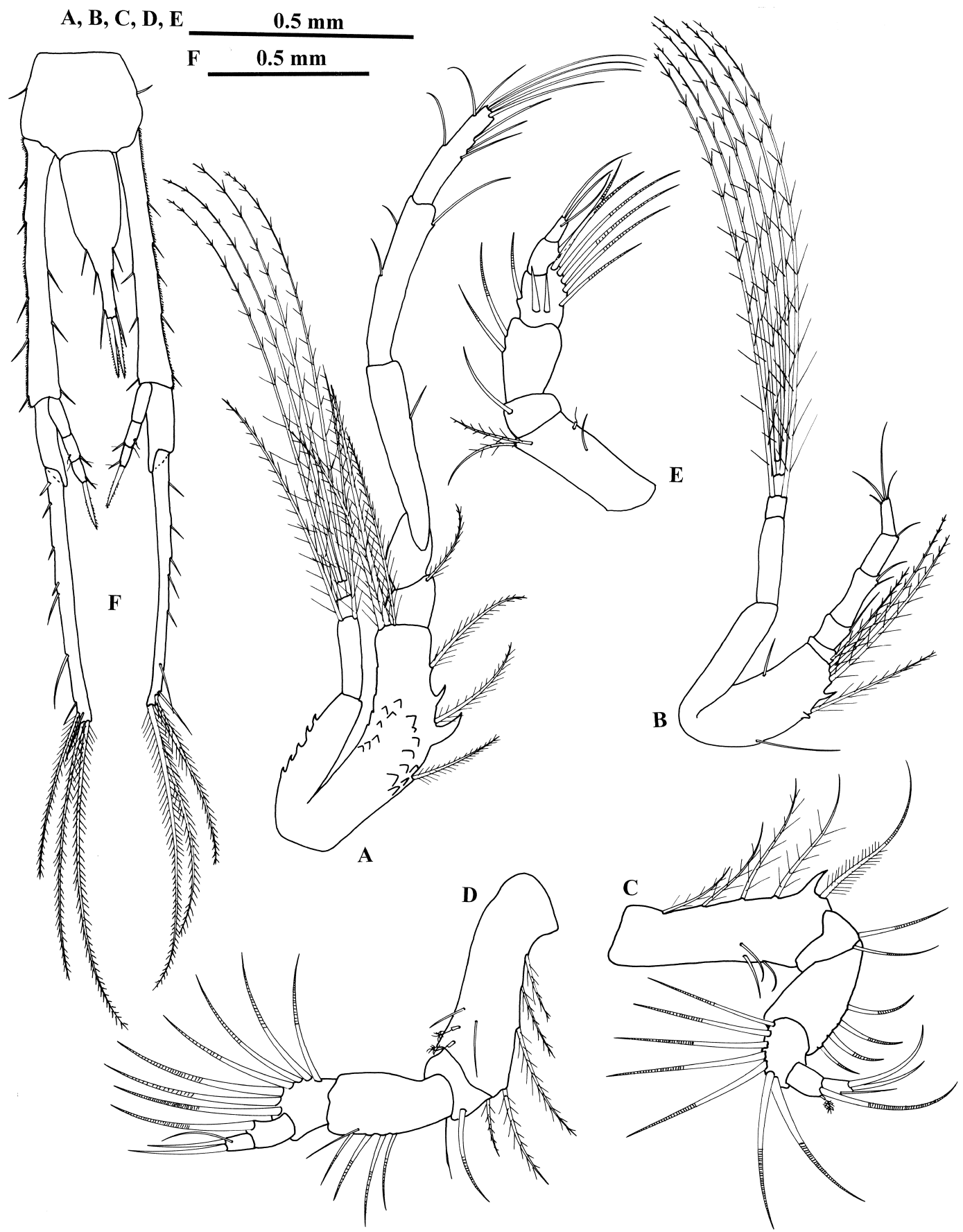

Fig. 5. Ektonodiastylis nimia ovigerous +. A, pereiopod 1; B, pereiopod 2; C, pereiopod 3; D, pereiopod 4; E, pereiopod 5; F, telson and uropods.

marginally and 2 stout simple setae terminally; exopod not present (Fig. 5D).

Pereiopod 5 basis longer than next 2 articles together, posterior distal corner with 2 plumose setae, anterior margin bearing 1 pedunculate seta and 1 simple slender seta; ischium one-half length of merus, posterior margin bearing 1 annulate seta; merus poste- 
rior margin bearing 2 annulate setae; carpus two-thirds length of merus, anterior margin with 5 stout annulate setae; propodus one-half length and one-half width of carpus, bearing 1 stout annulate seta distally; dactyl not as long as propodus, bearing 1 slender simple seta marginally, 2 stout simple setae terminally (Fig. 5E).

Telson 1.8 times as long as pleonite 6 , preanal and postanal sections subequal, postanal section lateral margins bearing 3 pairs of slender setae, with 1 pair of long microplumose setae terminally (Fig. 5F).

Uropod peduncles longer than telson, margins lined with many fine short hairlike setae, lateral margin bearing 5 slender simple setae, medial margin bearing 3 slender simple setae; endopod 0.25 times length of exopod, of 3 articles, article 1 longest and unarmed, article 2 distomedial corner with 1 stout seta, distolateral corner bearing 1 pedunculate seta and 1 small simple seta, article 3 distomedial corner with 1 stout seta, bearing 1 long microplumose seta terminally, medial and terminal setae all with 1 setule subapically; exopod of 2 articles, article 10.2 times length of article 2, unarmed, article 2 lateral margin bearing 8 slender simple setae, with 3 very long plumose setae terminally (Fig. 5F).

\section{Ektonodiastylis robusta, new species}

Material Examined.-Holotype, subadult $\delta$ (CMNC 1998-0017), Foxe Basin, Repulse Bay, $66^{\circ} 28^{\prime} \mathrm{N} 86^{\circ} 16^{\prime} \mathrm{W}$, rock/sand/mud, 23 August 1961.

Diagnosis.-Pseudorostral lobes directed dorsally in subadult male, pereionite 4 directly dorsal to pereionite 3 , telson subequal to uropod peduncles, uropod exopod length 1.5 times endopod length.

Etymology.-The specific name robusta indicates that the individual is larger and more robust than E. nimia.

Description.-Subadult male. Length 5.1 $\mathrm{mm}$. Carapace 0.6 times as deep as long, anterior one-third of dorsal crest lined with teeth, 1 tooth below pseudorostral lobes, pseudorostral lobes bearing many long plumose setae, sharply directed dorsally, antennal notch oblique, carapace ventral margin lined with teeth; pereionite 1 free and not covered by carapace; pereionite 2 with row of teeth dorsally, 1 tooth on anteroventral margin; pereionites 3 and 4 oriented horizontally rather than vertically, pereionite 4 dorsal to pereionite 3 , each with few teeth on margins; pereionite 5 also oriented mostly horizontally, produced bluntly posteriorly (Fig. 1C).

Antennule extending beyond pseudorostral lobes, very stout; peduncle article 1 longer than article 2, distodorsal corner bearing 1 plumose seta; peduncle article 2 unarmed; peduncle article 3 longer than article 1, dorsal margin bearing 8 long plumose setae, ventral margin having 2 small simple setae; main flagellum of 6 articles, articles 4 and 5 bearing 1 simple seta distally, article 6 bearing many simple setae terminally; accessory flagellum of 4 articles, extending to middle of article 4 of main flagellum, with 3 simple setae terminally (Fig. 6A).

Antenna extending past posterior margin of carapace, this individual being subadult; peduncle article 4 slightly shorter than article 5 , bearing 5 plumose setae; article 5 unarmed; flagellum of 22 articles, increasing in length and decreasing in width distally, articles unarmed (Fig. 6B).

Maxilliped 3 basis longer than all other articles together, twice as broad as merus, medial margin produced as several stout teeth, medial margin bearing 6 plumose setae, ridge down center of article bearing several more plumose setae, lateral margin lined with many fine hairlike setae, distal margin bearing 4 long, stout plumose setae; ischium compressed, unarmed; merus medial margin produced as 1 stout tooth, distolateral corner bearing 1 long, stout plumose seta; carpus subequal to merus, medial margin produced as 1 tooth and bearing 1 long stout and 1 slender plumose setae, lateral margin bearing 2 slender setae distally; propodus longer than carpus, bearing 2 plumose setae medially and 1 plumose seta distolaterally; dactyl with 3 simple setae marginally, 2 long simple setae and 1 microserrate seta terminally; exopod as long as basis, ischium, and merus together, basal article bearing few setae, flagellum bearing 10 long, stout plumose setae (Fig. 6C).

Pereiopod 1 basis as long as next 4 articles together, anterior, posterior, and distal margins lined with plumose setae, posterior margin produced as 1 tooth, center of article also produced as 1 tooth; ischium one-half 


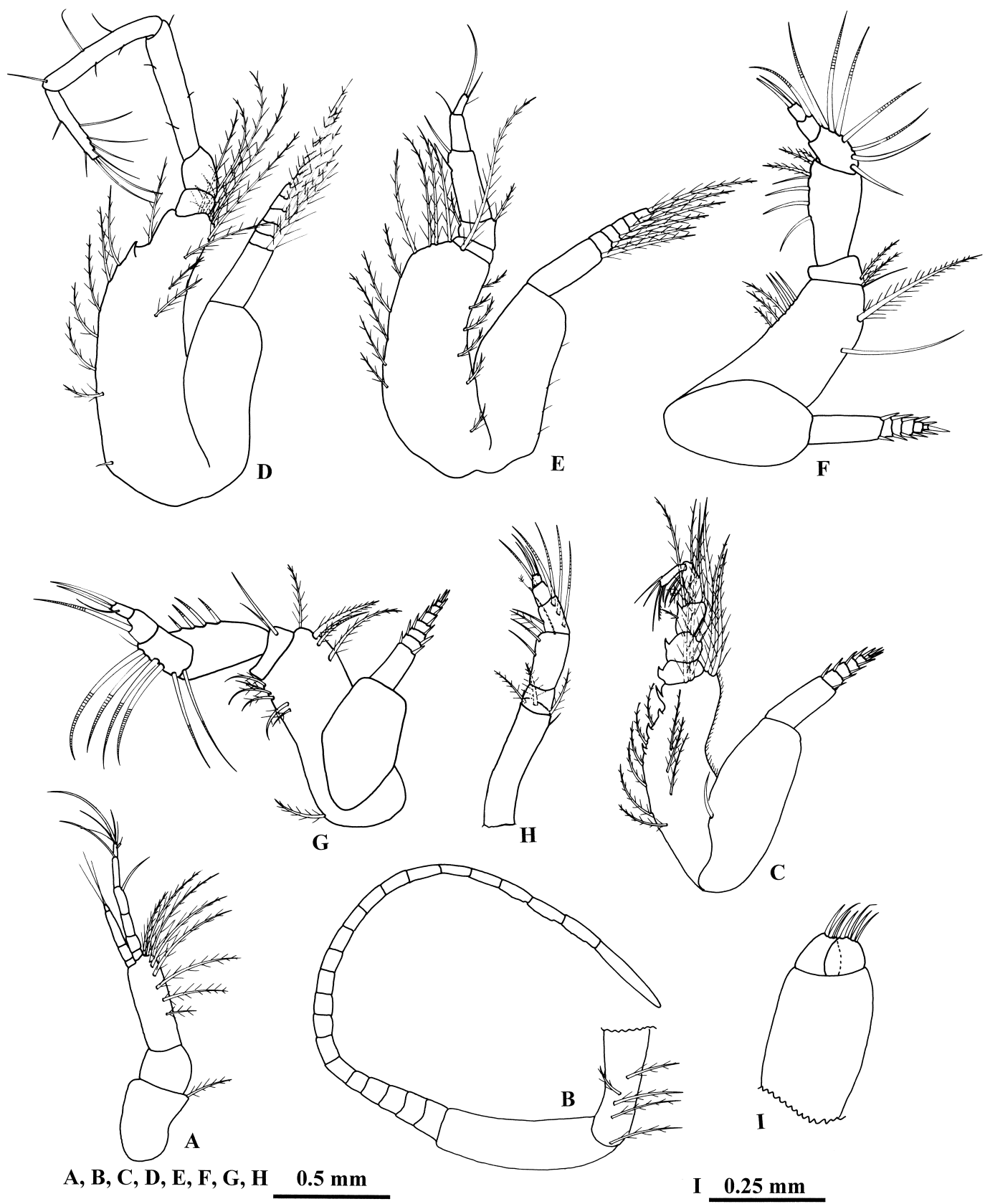

Fig. 6. Ektonodiastylis robusta holotype subadult ô. A, antennule; B, antenna; C, maxilliped 3; D, pereiopod 1; E, pereiopod 2; F, pereiopod 3; G, pereiopod 4; H, pereiopod 5; I, pleopod.

merus length, unarmed; merus unarmed; carpus and propodus subequal, bearing few short simple setae marginally; dactyl not as long as propodus, bearing 6 slender simple setae marginally, 2 stout simple setae terminally; exopod as long as basis, ischium, merus together, basal article armed with few small simple setae, flagellum bearing 12 long, stout plumose setae (Fig. 6D).

Pereiopod 2 basis expanded laterally, longer than all other articles together, 3 times as broad as carpus, anterior, distal, and posterior margins lined with plumose setae; ischium compressed, unarmed; merus twice as 


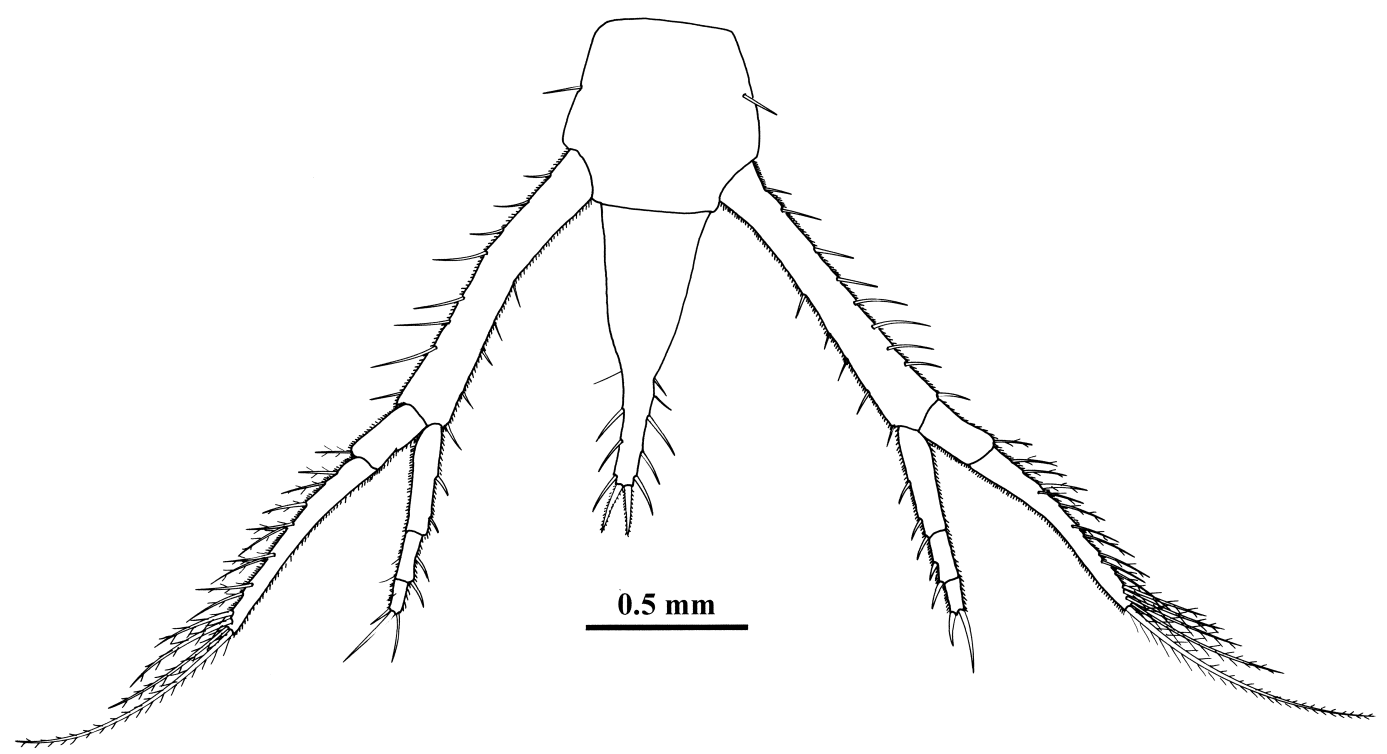

Fig. 7. Ektonodiastylis robusta holotype subadult ${ }^{2}$. Telson and uropods.

long as ischium, bearing 2 plumose setae distally; carpus 3 times as long as merus, posterior margin with 2 simple setae distally; propodus one-half length of carpus, bearing 1 simple seta distally; dactyl one-half as long as propodus, bearing 1 long and 4 simple setae terminally; exopod slightly shorter than entire endopod, basal article bearing few small simple setae, flagellum bearing 10 long, stout plumose setae (Fig. 6E).

Pereiopod 3 basis as long as all other articles together, posterior and anterior margins bearing plumose setae distally; ischium onehalf merus length, posterior margin bearing 3 sparsely plumose setae; merus posterior margin with 3 plumose and 4 annulate setae; carpus one-half length of merus, anterior margin bearing 7 stout annulate setae, increasing in length distally; propodus one-half length of carpus, with 1 stout annulate seta distally; dactyl not as long as propodus, bearing 1 slender simple seta marginally, 2 stout simple setae terminally; exopod longer than basis and ischium together, basal article armed with several plumose setae, flagellum bearing 12 setae (Fig. 6F).

Pereiopod 4 basis as long as next 3 articles together, anterior and posterior margins bearing plumose setae distally; ischium 0.14 times basis length, bearing 2 simple setae; merus 4 times ischium length, posterior margin having 4 annulate setae distally; carpus slightly shorter than merus, anterior margin bearing 6 long stout annulate setae, increasing in length distally; propodus one-half carpus length, bearing 1 stout annulate seta distally; dactyl shorter than propodus, with 1 slender simple seta marginally and 2 stout simple setae terminally; exopod longer than basis and ischium together, basal article unarmed, flagellum bearing 12 setae (Fig. 6G).

Pereiopod 5 basis as long as all other articles together, distal margin bearing 3 plumose setae; ischium unarmed; merus posterior margin bearing 1 plumose, 1 simple, and 2 annulate setae; carpus anterior margin having 4 annulate setae, 1 slender simple seta; propodus one-half length of carpus, bearing 1 stout annulate seta distally; dactyl shorter than propodus, bearing 1 slender simple seta marginally and 2 stout simple setae terminally (Fig. 6H).

One pleopod pair present, biramous, not fully developed (Fig. 6I).

Telson 1.5 times length of pleonite 6 , preanal and postanal sections subequal; postanal section bearing 4 or 5 slender setae on each side, 1 pair stout microplumose setae terminally (Fig. 7).

Uropod peduncles subequal to telson, margins lined with fine hairlike setae, lateral margin bearing 8 or 9 slender simple setae, medial margin bearing 5 slender simple setae; endopod two-thirds length of exopod, of 3 ar- 
ticles, all margins lined with fine hairlike setae, article 1 longest and bearing 3 stout setae medially, article 2 bearing 2 stout setae medially, article 1 with 2 stout setae terminally, all stout setae having 1 subapical setule; exopod of 2 articles, article 1 one-third length of article 2, bearing 1 slender seta distally, article 2 lateral margin bearing 12 or 13 sparsely plumose setae, with 2 very long plumose setae terminally (Fig. 7).

\section{DISCUSSION}

The two species placed within the new genus are clearly separable based on several characters. In E. nimia, the uropod exopod is at least twice the length of the uropod endopod, while in E. robusta, the uropod exopod is clearly longer than the endopod, but much less than twice the length of the endopod. The telson of E. nimia is shorter than the uropod peduncles, whereas the telson of $E$. robusta is distinctly longer than the uropod peduncles. In E. nimia, the pseudorostral lobes are nearly horizontal, whereas in E. robusta the pseudorostral lobes are directed distinctly dorsally. The carapace of E. nimia is less deep, relative to the length, than the carapace of $E$. robusta. In addition, the subadult male of E. robusta is in excess of $5 \mathrm{~mm}$ in length, whereas adult individuals of $E$. nimia are less than $4 \mathrm{~mm}$ in length.

The division of the Cumacea into its constituent families occurred at a time when few cumaceans were known from beyond the North Atlantic region. With the discovery of the gynodiastylids in the southern hemisphere, Stebbing (1912) distinguished the new family Gynodiastylidae from the more well-known Diastylidae on the basis of two major characters: absence of pleopods in the male and lack of an exopod on maxilliped 3 in the female in the Gynodiastylidae, whereas the Diastylidae possess two pairs of pleopods in the male and the female has an exopod on maxilliped 3. Additional species have slightly blurred the boundary between these two families: in the genus Atlantistylis, the male bears no pleopods but has an elongate antennule and an elaborate antenna, and in Paradiastylis the female does not have an exopod on maxilliped 3. Both genera are currently placed in the Diastylidae.

Ektonodiastylis fits more completely the current definition of the cumacean family Diastylidae, rather than the closely related fam- ily Gynodiastylidae. The male antennule of Ektonodiastylis extends beyond the carapace posterior margin and there is one pair of pleopods, whereas in male Gynodiastylidae the antennule typically does not extend beyond the carapace and pleopods are absent. The females of Ektonodiastylis possess an exopod on maxilliped 3, which is absent in female Gynodiastylidae. The telson, in both male and female, possesses distinct pre- and postanal sections, and the postanal section is armed with robust setae, including large terminal setae; in contrast, Gynodiastylidae typically do not possess clear pre- and postanal sections of the telson, and the telson is unarmed or armed with minute setae.

Ektonodiastylis seems clearly derivable from Brachydiastylis. Brachydiastylis has two normal pairs of pleopods while Ektonodiastylis has only a single pair. In addition, the adult male of Ektonodiastylis shows the same peculiarity in construction of the propodus and dactyl on pereiopod 2 seen in Brachydiastylis, except that the dactyl is greatly reduced. Otherwise, the two genera are nearly enough alike that Hansen, whose material did not include males, can be excused for including E. nimia in the same genus as B. resima.

\section{ACKNOWLEDGEMENTS}

The National Science Foundation (Grant DEB1521783) supported this work as part of the Partnerships to Enhance Expertise in Taxonomy (PEET) program. Thanks are due to the Zoological Museum in Copenhagen (ZMUC) and the Canadian Museum of Nature in Ottawa for access to their collections. One of us (SG) thanks Dr. Ole Tendahl, Anders Giessing, and Rikke Hansen for their hospitality while working in Denmark.

\section{LiTERATURE CiTED}

Bate, S. 1856. On the British Diastylidae.-Annals and Magazine of Natural History 17: 449-465.

Day, J. 1980. South African Cumacea, Part 4: Families Gynodiastylidae and Diastylidae.-Annals of the South African Museum 82: 187-292.

Hansen, H. 1920. Crustacea Malacostraca. 4. The Order Cumacea.-Danish Ingolf Expedition. Copenhagen 3(6): 1-86.

Lomakina, N. 1952. Cumacea of the seas of the USSR.-Opredeliteli po faune SSSR 66: 1-301. [In Russian.]

Stebbing, T. R. R. 1912. The Sympoda, Part 6.-Annals of the South African Museum 10: 129-176.

Schiotte, T. 1989. Marine Mollusca from Jørgen Brønlund Fjord, North Greenland, including the description of Diaphana vedelsbyae n. sp. Meddelelser om Grønlund.-Bioscience 28: 1-24.

RECEIVED: 7 December 1998

ACCEPTED: 12 May 1999. 